\title{
Impact of COVID-19 on mental health and occupational burnout in a surgical unit in Singapore
}

\section{Dear Editor,}

In this study, we assessed the impact of COVID-19 on the psychological well-being and burnout among staff who manage critically ill general surgery and trauma patients as part of the Acute Care Surgery (ACS) service at the Singapore General Hospital, Singapore. The ACS team may be exposed to COVID-19 during consultations, high-risk surgical procedures and endoscopy that may aerosolise the virus. Our assessment revealed a positive mindset among the respondents, and that protective factors are paramount to maintain positive well-being.

In response to the COVID-19 pandemic, the Singapore government escalated its Disease Outbreak Response System Condition level to Orange on 7 February 2020, triggering containment and mitigation strategies. ${ }^{1}$ The psychological sequelae of the pandemic deserve attention. ${ }^{2}$ Data from previous outbreaks show that healthcare workers have suffered from post-traumatic stress disorder, depression and substance abuse. ${ }^{3}$ In Wuhan, the initial epicentre of COVID-19, healthcare workers faced enormous challenges such as increased risks of infection and transmission of the disease to patients and family, and working with inadequate supply of personal protective equipment (PPE). ${ }^{4}$

The rapid surge of cases in Singapore has increased the workload and pressure placed on our healthcare staff. Few studies have addressed these issues outside of China during the current pandemic..$^{5-7}$

This study was approved by the Institutional Review Board. It was a single-centre, cross-sectional study conducted via an online questionnaire (Qualtrics, Provo, US), accessible on personal computers and mobile devices. Doctors at all levels of seniority in the ACS service were invited to participate 2 months after the first case of COVID-19 in Singapore (23 January 2020). The questionnaire aimed to collect the respondents' subjective assessments of fear, anxiety, depression and burnout during this period. All responses were anonymised and results were aggregated before analysis.

The questionnaire comprises 4 parts that collected (1) demographic data, (2) perceived health status, (3) perceived risk of COVID-19 exposure in relation to workplace safety and contact with patients suspected or confirmed with COVID-19, and (4) impact on surgical training and personal development. Consolidated data for (2), (3) and (4) in Fig. 1 present the respondents' self-assessment of their psychological well-being and syndromes of burnout that they have experienced.

The Maslach Burnout Inventory (MBI) is a wellvalidated psychological tool used in research on burnout in various occupations and workplaces. ${ }^{8,9}$ The MBIHuman Services Survey for Medical Personnel (MBIHSS (MP)) was designed and adapted to assess burnout in healthcare workers. It comprises 22 statements to assess 3 burnout syndrome domains: emotional exhaustion, de-personalisation and personal accomplishment.

Nine out of 22 items from the MBI-HSS (MP) were selected for this study based on relevance and relatability. Respondents indicated their level of agreement/disagreement with each statement via a 5-point Likert scale.

The data were tabulated using Qualtrics and further analysed using Microsoft Excel version 2013 (Microsoft Corp, Redmond, US). Responses for agree/strongly agree and disagree/strongly disagree were coalesced for ease of reporting.

The survey response rate was $98 \%$ (90/92 respondents) of doctors who worked in the ACS team from February to March 2020. The majority of the respondents (59\%) were junior doctors comprising house officers, both medical officers and residents who were unmarried (71\%) with no dependants living in the same household (63\%).

Shown in Fig. 1, more than $80 \%$ of respondents agreed with the positive statements that reflect fulfillment and satisfaction at work. Notably, $100 \%$ of respondents enjoyed working within their teams, and $83 \%$ expressed confidence in handling unexpected events that may occur at and outside of work-in the context of the pandemic, significant events outside of work include being quarantined, having a loved one contract COVID-19, closure of important services and a nationwide lockdown.

Less than $12 \%$ of respondents agreed with the negative statements that assessed for energy depletion or exhaustion, increased mental distance from work or feelings of negativism, and reduced professional efficacy. No respondents expressed apathy towards the care of their patients. 
Fig. 1. Response to statements pertaining to (A) psychological well-being and occupational burnout, (B) COVID-19 exposure and $(\mathrm{C})$ impact on surgical training and personal development.

(A)

(B)

(C)



ACS: Acute Care Surgery; SRs: senior residents

Numbers indicate percentages.

One third $(33 \%)$ of respondents were concerned that they may contract COVID-19 and 57\% were concerned that their loved ones would be at higher risk of COVID-19 exposure as a result of their work.

However, the majority of respondents agreed that the ACS system promoted safe segregation among healthcare workers during the COVID-19 pandemic $(67 \%)$ and there were appropriate measures to ensure they were adequately protected (96\%). Having dependants living in the same household was associated with a higher concern for COVID-19 exposure compared to those with no dependants at home (70\% vs $49 \%$ ). Most respondents also agreed that the ACS system allowed junior staff to better keep to duty hour regulations (84\%), better attend to personal needs outside of work $(80 \%)$ and achieve better work-life balance $(78 \%)$. Eighty-nine percent agreed that the number of calls they perform each month is manageable, $78 \%$ agreed 
that they had at least 1 day free from all clinical responsibilities before their next call, and $67 \%$ agreed that they have never exceeded 30 hours of continuous on-site duty when they were on call despite increased workload and manpower demands during the COVID-19 pandemic. There were no significant differences between responses from junior and senior staff members.

The majority of respondents had adequate time for academic activities and self-learning (72\%), and despite the postponement of elective non-cancer surgeries, $79 \%$ of respondents agreed that there remained adequate case volume for learning.

As the pandemic progresses, anxiety, depression and stress regarding workplace safety can intensify and lead to deteriorations in mental health given concerns of PPE shortages, loneliness and separation from loved ones. ${ }^{5,10,11}$ Therefore, it was encouraging that $96 \%$ of respondents felt adequately protected from COVID- 19 . The ACS team was able to observe social distancing by limiting visits to the emergency department to review unstable patients, cancelling elective surgery and clinics, and designating wards for patients infected/suspected with COVID-19, together with ramped-up cleaning. Helplines for confidential mental health advice were made available. Support from the Singapore government ensured sustained supply of PPE, powered air-purifying and N95 respirators, masks, face-shields, hair nets and gloves. Thus, safety of the staff was not compromised. A recent meta-analysis supported our findings that reduced psychological morbidity for healthcare workers was associated with clear communication, access to PPE, adequate rest and availability of psychological support. ${ }^{12}$ Other systemic measures include identification of groups that are at high risk of psychological distress, early psychological screening and prompt intervention using cognitive or mindfulness-based behavioural therapy. ${ }^{13}$

Although reassuring to see few respondents had expressed feelings of frustration, emotional drainage, callousness or fatigue from their work, long-term assessment is required to assess the strength of these findings, following reported prevalence of $50 \%$ burnout rate such as those in general surgery. ${ }^{14}$

The main strength of this study was the near complete response that bolstered its internal validity. However, as this is a single-centre study performed at a single time point with a small sample size, it may not be representative of other trauma and acute surgical units, and long-term studies are needed to ascertain if the positive findings persist with progression of the pandemic. Nonetheless, we have found that the following protective factors are paramount in maintaining positive well-being of our population-availability of PPE, appropriate hospital safeguards, team structure to limit exposure and crossinfection, safe working hours and efforts to provide reward for those at the frontline.

\section{REFERENCES}

1. Chen JI, Yap JC, Hsu LY, et al. COVID-19 and Singapore: From Early Response to Circuit Breaker. Ann Acad Med Singap 2020;49:561-72.

2. Adams JG, Walls RM. Supporting the health care workforce during the COVID-19 global epidemic. JAMA 2020;323:1439-40.

3. Brooks SK, Webster RK, Smith LE, et al. The psychological impact of quarantine and how to reduce it: rapid review of the evidence. Lancet 2020;395:912-20.

4. Wang J, Zhou M, Liu F. Reasons for healthcare workers becoming infected with novel coronavirus disease 2019 (COVID-19) in China. J Hosp Infect 2020;105:100-1.

5. Chong MY, Wang WC, Hsieh WC, et al. Psychological impact of severe acute respiratory syndrome on health workers in a tertiary hospital. Br J Psychiatry 2004;185:127-33.

6. Lai J, Ma S, Wang Y, et al. Factors associated with mental health outcomes among health care workers exposed to coronavirus disease 2019. JAMA Netw Open 2020;3:e203976.

7. Chew NWS, Lee GKH, Tan BYQ, et al. A multinational, multicentre study on the psychological outcomes and associated physical symptoms amongst healthcare workers during COVID-19 outbreak. Brain, Behav Immun 2020;88:559-65.

8. Maslach C, Schaufeli WB, Leiter MP. Job burnout. Annu Rev Psychol 2001;52:397-422.

9. Maslach C, Jackson SE, Leiter MP, et al. Maslach burnout inventory. Palo Alto: Consulting Psychologists Press; 1986;21:3463-4.

10. Kim JS, Choi JS. Factors influencing emergency nurses' burnout during an outbreak of middle east respiratory syndrome coronavirus in Korea. Asian Nurs Res (Korean Soc Nurs Sci) 2016;10:295-9.

11. Tan BYQ, Chew NWS, Lee GKH, et al. Psychological impact of the COVID-19 pandemic on health care workers in Singapore. Ann Intern Med 2020;173:317-20.

12. Kisely S, Warren N, McMahon L, et al. Occurrence, prevention, and management of the psychological effects of emerging virus outbreaks on healthcare workers: rapid review and meta-analysis. BMJ 2020;369:m1642.

13. Ho CS, Chee CY, Ho RC, et al. Mental Health Strategies to Combat the Psychological Impact of COVID-19 Beyond Paranoia and Panic. Ann Acad Med Singap 2020;49:155-60.

14. Dimou FM, Eckelbarger D, Riall TS. Surgeon burnout: a systematic review. J Am Coll Surg 2016;222:1230-9.

Beatrice Fangju Koh, ${ }^{1}$ FRCS, Mingzhe Caii, ${ }^{1}$ MMed (Surgery), Stephanie Cheng, ${ }^{1}{ }_{M B B S}$, Jeremy Chung Fai $\underline{\mathrm{Ng}},{ }_{F R C S}$, Sachin Mathur, ${ }^{1}$ FRACS

${ }^{1}$ Department of General Surgery, Singapore General Hospital, Singapore

Correspondence: Dr Mingzhe Cai, Department of General Surgery, Singapore General Hospital, Outram Road, Singapore 169608.

Email: mingzhe.cai@u.nus.edu 